\title{
Correspondence
}

\section{When not to do a lumbar puncture}

Sir,

Although an important point is made that a lumbar puncture may not be the essential first investigation for a very sick child who may have meningitis, I think there are some points in Dr Addy's annotation which deserve further discussion. ${ }^{1}$ I think it is unfortunate to use the word 'assault' about this investigation. Also the fact that cerebral oedema and 'coning' are quite commonly found in children dying of meningitis does not necessarily indicate that lumbar puncture contributed to their death.

There are, I think, three different situations which this annotation is referring to and there are quite different reasons for omitting or deferring a lumbar puncture in each.

(1) In, for example, a child with classical clinical presentation of meningococcal infection a lumbar puncture may be merely unnecessary and could delay, though of course should not, the first dose of antibiotic.

(2) The child may be 'too sick for lumbar puncture.' This, I think, is the main point of the article. I think it may be clearer to say that, for a child in coma, there are priorities in resuscitation and investigation which may cause one to defer a lumbar puncture until problems of underventilation, status epilepticus, circulatory failure, and hyperpyrexia have been dealt with. There can be no doubt that dealing with underventilation and presumed cerebral oedema are much more important. Very often it takes only about half an hour to have either completed the above or at least to have made progress, and a lumbar puncture may then be safely performed. There is absolutely no reason why antibiotics should not be given for presumed meningitis at the outset of resuscitation. If the child's depth of coma warrants this, then intracranial pressure monitoring by an intraventricular drain does, of course, provide cerebrospinal fluid as well.

As an additional point about cause of death, it has been our experience that, in children with overwhelming infection, a major problem has often been failure of adequate cardiac output, which fails to respond to drug treatment or our efforts at minimising the effects of cerebral oedema. (3) The danger of delay in the diagnosis of other conditions is another consideration. We see a large number of children with conditions which have been suspected of being meningitis, including tumours, abscesses, vascular episodes, and unusual presentations of epilepsy, in which the lumbar puncture is decisive in early referral. One could say that the lumbar puncture was a mistake and that the children would have been better referred for an urgent computed tomogram. I fear, however, that if the habit of not doing a lumbar puncture caught on, that a number of these children's diagnoses would be further delayed.

\section{Reference \\ 1 Addy D. When not to do a lumbar puncture. Arch Dis Child 1987;62:873-5.}

B G R Neville Newcomen Centre, Guy's Hospital,

St Thomas Street,

London SE1 9RT

Sir,

We have followed the correspondence debating the need for lumbar puncture in the diagnosis and management of meningitis with interest, but wish to express our concern regarding the recent annotation on the subject. ${ }^{1}$ Nowhere is consideration given to the difficulties surrounding the diagnosis of tuberculous meningitis. In Third World countries tuberculous meningitis remains a commonly encountered form of meningitis in childhood ${ }^{2}{ }^{3}$ but the diagnosis should be considered at all times in every setting. ${ }^{4}$

Early diagnosis is crucial, as the outcome is determined by the stage of the disease at which treatment is started. ${ }^{5}$ Many children show subtle premonitory symptoms before those of meninigitis appear, and the clinician may be astute enough to perform a lumbar puncture at this optimal stage. Other children, however, present acutely with a picture indistinguishable from meningitis due to other causes. Without a knowledge of the results of cerebrospinal fluid examination it is difficult to imagine how the clinician is going to consider the possibility of tuberculous meningitis in the differential diagnosis. A policy of diagnosing meningitis on clinical grounds, initiating meningitis treatment, and awaiting clinical improvement will be disastrous, if not fatal, in the case of this disease.

It is true that in a minority of cases the presence of a strong family history, or a chest radiograph showing pulmonary tuberculous in the presence of typical neurological signs, enables a confident diagnosis to be made. It is also true that when diagnostic uncertainty exists, computed tomography may assist the diagnosis of tuberculous meningitis by showing the characteristic basal exudate and associated hydrocephalus." In most countries where tuberculous meningitis is a common problem, however, facilities are limited, and even in developed countries a critical period may pass before computed tomography is undertaken.

We would entirely agree that lumbar puncture is both dangerous and unnecessary in a child with meningococcal septicaemia, and in cases with definite signs of raised intracranial pressure. But in developing countries, or in any country where tuberculous meningitis is still encountered, a policy of not carrying out lumbar puncture in cases of meningitis is in our opinion a recipe for disaster. 


\section{References}

1 Addy DP. When not to do a lumbar puncture. Arch Dis Child 1987;62:873-5.

2 Donald PR, Burger PJ, Becker WB. Paediatric meningitis in the Western Cape. A three year hospital-based prospective study. $S$ Afr Med J 1986;70:391-5.

3 Deeny JE, Walker MJ, Kibel MA, Molteno CD, Arens LJ. Tuberculous meningitis in children in the Western Cape. Epidemiology and outcome. $S$ Afr Med J 1985;68:75-8.

+ Naughten E, Weindling AM, Newton R, Bower BD. Tuberculous meningitis in children. Recent experiences in two English centres. Lancet 1981;ii:973-5.

5 Arens LJ, Deeny JE, Molteno CD, Kibel MA. Tuberculous meningitis in children in the Western Cape: neurological sequelae. Paediatric Rev Commun 1987:1:257-75.

6 Bhargava S, Gupta AK. Tandon PN. Tuberculous meningitisa CT study. Br J Rad 1982;55:189-96.

P R Donald

Department of Paediatrics and Child Health,

University of Stellenbosch, South Africa

M A KIBEL

Institute of Child Health,

University of Cape Town, South Africa

Correspondence now closed.

\section{Management of children with head injuries in district general hospitals}

Sir,

In his paper on the 'management of children with head injuries in district general hospitals' Mr Hayward expresses the view that the primary responsibility for children with head injury should be undertaken by paediatricians. ${ }^{\prime}$ We agree with this opinion but would like to highlight that this does not appear to be current practice.

We have just completed a survey of all children who were admitted with or who died from a head injury in the Northern Region between the years 1979 and 1987. In this period there were 25152 such children of whom $258(1 \%)$ died. Thirty three children died at the site of the accident and a further 106 died on their way to hospital.

Altogether 25009 children were admitted to hospital; $10561(42 \%)$ of these children were admitted under the care of specialists other than a paediatrician or neurosurgeon. The number of children admitted to a regional hospital with expertise in both paediatrics and neurosurgery was 7179 , and $6246(87 \%)$ of these children were cared for by either a paediatrician or a neurosurgeon or there was shared care. The number of children admitted to a district general hospital was 17830 , of whom $8202(46 \%)$ were cared for by a paediatrician; $9628(54 \%)$ of the children admitted to the district general hospitals were cared for by other specialists, primarily general or orthopaedic surgeons.

Of the 116 children admitted to hospital and who subsequently died, $33(28 \%)$ died at a district general hospital. Twenty seven of these 33 children died under the care of a general or orthopaedic surgeon. Twenty four of these 33 children survived more than six hours and could therefore have been transferred to a specialist centre.

Head injury is the most common cause of death and acquired disability in childhood, yet there is no general agreement as to the most effective approach to either acute management or to rehabilitation. The relative paucity of research into specific aspects of the management of children with head injury may in part reflect the present wide spread dispersal of the care of these children among different specialties.

We agree with Mr Hayward that the high incidence of learning and behavioural difficulties which may follow even mild head injury ${ }^{2}$ emphasises the importance of paediatric involvement in the acute and long term care of all head injured children even those with mild head injury.

We believe that there is an urgent need for paediatricians to revise the facilities offered to children with head injury to ensure that they receive the highest standard of care. Furthermore, research is needed in both the acute management of children after head injury and their rehabilitation in an attempt to reduce the high mortality and morbidity.

\section{References}

1 Hayward R. Management of children with head injuries in district general hospitals. Arch Dis Child 1987;62:1283-6.

2 Klonoff $H$, Low MD. Clark C. Head injuries in children; predisposing factors. Am J Public Health 1977;61:2405-11.

P M Sharples, A Aynsley-Green, and J A Eyre Department of Child Health, University of Newcastle upon Tyne

\section{Tight nuchal cord and neonatal hypovolaemic shock}

Sir,

Dr Vanhaesebrouck and his colleagues do well to warn of the danger of the hypovolaemic shock that may follow clamping and division of a nuchal umbilical cord. 'During studies in Bristol (1963-5), it was observed that there was normally a shift in blood volume from the fetus to the placenta during the second stage of labour. This 'fetoplacental transfusion' was attributed to the more ready compression of the soft walled, low pressure umbilical vein than the firm, high pressure umbilical arteries, between the body of the fetus and the wall of the birth canal. When the umbilical cord was clamped at the moment of birth, as much as $30-40 \%$ of the normal fetal blood volume might be trapped within the placental vasculature rendering the placental congested, tense, bulky, and more likely to be retained.

On the other hand the newly born infant often exhibited transient signs of hypovolaemic shock with hypotension, tachycardia, and pallor due to peripheral vasoconstriction. While this insult was tolerated remarkably well by most healthy term infants, it was capable of causing resuscitative and adaptive problems among those that were preterm, 